\title{
Studying sediment transport dynamics by using the Smart sphere
}

\author{
Zaid Alhusban $^{1}$, Manousos Valyrakis ${ }^{2}$ \\ ${ }^{1}$ PhD graduate, School of Engineering, University of Glasgow, UNITED KINGDOM \\ ${ }^{2}$ Manager of the Water Engineering Lab, University of Glasgow, UNITED KINGDOM
}

z.al-husban.1@research.gla.ac.uk

\begin{abstract}
A$ new method is introduced by using high precision accelerometer and gyroscope micro-electromechanical sensors (MEMS), which can record Lagrangian observations of sediments and shed light into the dynamics of sediment transport processes at above threshold conditions. The sensor can be used under a range of well-controlled flow conditions and can record measurements at high frequency $(200 \mathrm{~Hz})$, which can be used at the field. The smart sphere performance was evaluated by comparing its rotation and acceleration readings from the sensors to the video recordings of both top and underwater high-speed camera for a range of flow rates and sphere densities. Furthermore, an initial attempt to compare the smart-sphere's velocity is achieved, by transforming the particle's velocity from the Lagrangian frame of reference, obtained from the inertial sensor, to its velocity at the Eularian frame, obtained from the top camera.
\end{abstract}

Keywords: Lagrangian, high frequency, velocity, micro-electomechanical sensors.

Received: 10/05/2020 - Accepted: 12/06/2020

\section{Introduction}

For several decades, many theoretical and experimental studies were devoted to increase the understanding of the mechanics responsible for transporting sediment as they are center of attention for many hydraulic researchers, geomorphologists and industrial projects. Measurements of sediment motion have been primarily limited to indirect observations with acoustic and optical instruments. Radio frequency identification tags implanted in natural rounded riverbed spheres were used to study sediment transport, but they have limited capacity to detail exact sphere movement. Smart loggers have been developed inside suitable sensors to store data and determine the actual paths of individual grains [1]. The approach after analysis had many technical challenges in attaining and storing the data $[2,3]$ after calibrating the sensor tried to get accurate information about the entrainment conditions of a sensor, but even though the analysis result of the data does not permit the precise resolution of the smart sphere path. Recent technological advancements in the area of sensing and instrumentation has received increasing attention and rendered it possible to record Lagrangian observations of sediments and shed light into the dynamics of sediment transport processes, at an unprecedented resolution and accuracy by using high precision accelerometer and gyroscope sensors micro-electomechanical sensors
(MEMS)[4]. The MEMS sensor can record inertial dynamics such as accelerations $\left(\mathrm{m} / \mathrm{s}^{2}\right)$ (related to the near bed hydrodynamic forces acting on it), angular velocities $(\mathrm{rad} / \mathrm{s})$, magnetic field and hence forces and energy. This sensor will be capable of higher frequency (up to approximately $200 \mathrm{~Hz}$ ) and have enhanced data acquisition and storage so it can provide a novel tool for investigating sediment transport dynamics. The "smart sphere" is a contemporary sensing equipment under development which is miniaturized to scales that enable implementation of dynamic micro-sensors within natural sediment spheres (of diameter $<80 \mathrm{~mm}$ ), so it can mimic the behaviour of a naturally rounded sediment particlesand determine the full inertial dynamics of sediment transport in 3D space from the moving bodyframe of individual spheres, giving an insight on how individual grains experience transporting forces during their movement from the riverbed. The purpose of this research project is to demonstrate how such technologies can improve our understanding of sediment transport dynamics . The theory and previous research of sediment transport will be considered in order to develop a better understanding of the problem and design suitable experiments.Sphere transport experiments under a range of well-controlled, fully turbulent and rough flow conditions are conducted in a flume at the Water 
Engineering Lab, of the University of Glasgow, to study the sediment transport above the threshold of motion (between the threshold of motion and below continuous transport) for the smart sphere. It is expected in all experiments that the effect of hydrodynamic forces on the smart sphere is comparable to their effects on real sediment particle transporting in riverbeds. he equipment used to collect the data will be described, along with the procedures used to obtain and analyse the results. The findings will be further discussed, and the 'smartsphere's' capacity to study the dynamics of sphere motion, as well as its use as a novel tool for the practioners and hydraulic engineers will be assessed.

Sediment transport of coarse bed material is very complex process getting a probabilistic nature because of the intermittent character of turbulent flow forcing, local arrangement properties and the randomness of sphere. For this reason a principle that is universally accepted and strongly describes this dynamical process does not yest exist. The criteria generally employed for engineering applications, such as Shields shear stress, is averaged in time and space with high level of variability, so it fails to capture the dynamic character of hydrodynamic forcing leading to sediment transport. Lately, novel sediment transport criteria using a dynamic defintion that highlights the importance of adequate energetic turbulent flow structurs have been suggested [ 5 , 6]. In contrast with former studies based on the mean averaged static force balance approaches. Therefore, it is assumed that for the same mean flow velocity the sphere will be subject to different forces and responses. The application of these criteria in engineering practice remains relatively low even though these criteria can capture the fully dynamic nature of sphere movement in turbulent flows. The research attempts to cover the gap in calculating the sediment transport by examining an application of a novel approach and taking advantage of recent technological advances that offer high precision sensors and microelectronic mechanical systems (MEMS), which could be used to enable approaching flow induced bed material transport dynamics from a Lagrangian perspective, to improve the understanding of mechanism of bed load transport and the processes involved in the near bed region of a turbulent open channel flow by measuring inertial dynamics at scales suitable for gravel-sized sediment, because the miniaturization of sensing equipment has made the sensor capable of measuring inertial dynamics such as acceleration and angular velocity) feasible. This advanced low-cost tool can be used in a lot of applications after calibrating and testing the sphere carefully in controlled flow conditions and can be transformative for the water engineering industry. The results of this study could lead to a better understanding of sphere motion dynamics at above the sediment transport thresholds during its movement from the riverbed.

\section{Experimental investigation}

The experiments for this study were conducted to evaluate the performance of the "smart- sphere" at the Arm field flume in the Water Engineering Lab at the University of Glasgow where conditions above Threshold were created in a high capacity research flume, and the responses of a custom-made instrumented sphere fitted with inertial measurement sensors, namely the 'smart-sphere', sitting in a local topography of known geometry were observed. The sphere's movements were monitored using both the video input from a high-speed camera and the data from sensors fitted in the sphere . Flow measurements were recorded using a 3D Acoustic Doppler Velocimeter (Nortek vectrino 1). Figure 1 shows the internal components of the instrumented particle

\section{II.1 Description of the smart sphere}

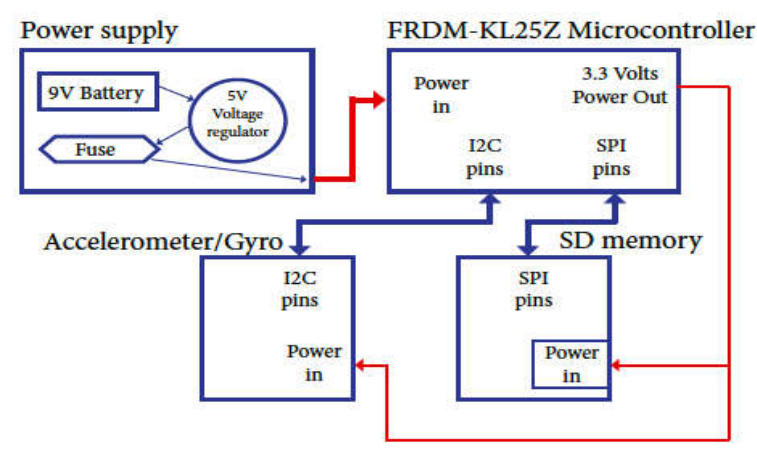

Figure 1. The internal components of the instrumented particle

As a result, the sensor can resolve 9 Degrees of Freedom for the movement of the unit. The data are recorded at regular intervals and stored on the external memory module via a microcontroller, embedded in a 3D-printed waterproof hollow sphere [7]. The instrumented board is appropriately fit while keeping the acceloremeter and gyroscope on the centre of the sphere, to achieve a nearly uniform distribution of the mass, which could otherwise may bias its motion. The gyroscope has excellent ability of measuring angular motions, but it lacks an absolute orientation reference in contrast with the rest sensors as the axes of the sensor are not aligned to the axis of the local Eulerian frame and they are taken with respect to the body frame, so the same vectors will be recorded differently in the frame of the sensor compared to their initial form in the Eulerian frame [8]. The measurements should be corrected to avoid possible effects of signal 
drifts. Therefore, readingsfrom the magnetometer and the acceleroter, which measures magnetic direction, are used to form an absolute component of orientation that corrects any short-term drifts made by the gyroscope.The embedded sensors have a sufficiently high sampling rate $(200 \mathrm{~Hz})$ to measure angular velocities and accelerations changes undergone by the smart sphere in our experiments. Time series of the sphere response, data from the gyroscope and the accelerometer readings were stored in the sensor system's memory. These data were post-processed offline using an appropriate scientific programming language (Matlab) which gives the ability to measure for the first time the inertial dynamics such as 3-dimensions local accelerations $\left(\mathrm{m}^{2} / \mathrm{s}\right), 3$-dimension angular velocities ( $\mathrm{rad} / \mathrm{s})$ from angular acceleration, and relate these to the near bed hydrodynamic forces acting on it and under threshold flow conditions. Figure 2 shows the Time and acceleration relationship for one of the trials. After that the total acceleration magnitude was calculated as follows:

$$
a=\sqrt{a_{x}^{2}+a_{y}^{2}+a_{z}^{2}}
$$

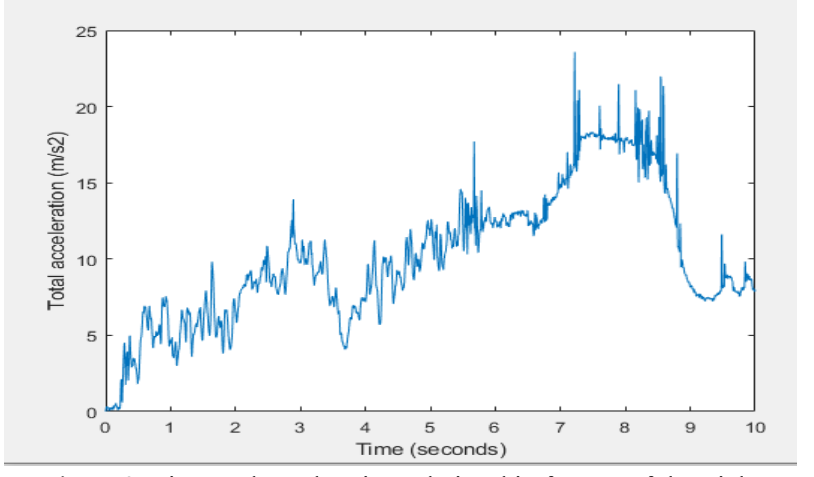

Figure 2. Time and acceleration relationship for one of the trials

The accelerometers accumulate errors in measurements, and they exhibit drift, no uniformity, misalignment, bias, sensitivity (due to interference, temperature, etc).

Then post-processing will be done to the raw data extracted from the sensors to correct any errors in the acquired signal by using 3 steps moving average for the acceleration data, which is used as a filter to remove noise from data. The "smart-sphere" employed is a custom-made instrumented sphere with $6.5 \mathrm{~cm}$ diameter with a built-in sensor for acceleration and rotation measurements. Figure 3 shows the smart sphere with its internal components.

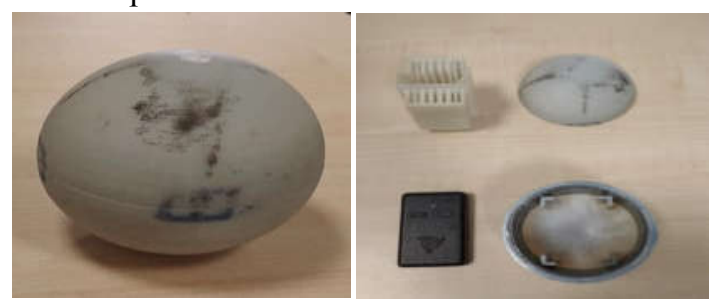

Figure 3.a) The smart sphere shell b) The smart sphere with its internal components: inner casing, outer casing, sensor, O-ring, holder for sensor and density control

Smart spheres are made of plastic material and deployed in natural environments, such as rivers, are affected by large forces and damaging environmental conditions. It is important to ensure that the electromechanical components of the sensor will not be stressed extremely and will remain dry during operation to avoid affecting sensor data results. The encasement in the smart sphere is required to measure large forces experienced by mobile river sediment grains. The sensors are housed within two separate conjoined cases that fit the sensor, hold it in place and keep it operational. The smart sphere is manufactured where sensor should be in the middle of sphere to get reasonable results and placed with known initial orientation. Even though the size, specific density, shape and material are not matching the physical natural sphere, the instrumented sphere is used because it takes surrogate measurements, which can relate to transport metric. So, it is good to study the process (mode of transport) and physics. Moreover, the results will not be exact but expected to correlate to actual same transport rates. Having the sensor at the centre of smart sphere, surrounded by equally distributed weights, a uniform distribution of mass was obtained with uniform smart sphere, so that the motion was not biased and offsets in the readings were minimized. The smart sphere was powered by an independent power supply to ensure autonomy and sufficiently long periods of operation, appropriate for its future deployment in the field. The unit was powered by a battery, which generally powers the circuit up to about 50-60 minutes continuously. The casing as shown in Figure 5 is designed to accommodate different quantities of weights, thus having an adjustable density of $500-2900 \mathrm{~kg} / \mathrm{m}^{3}$. The casing designs were produced using appropriate software (SolidWorks TM) and where built using a rigid opaque photopolymer material and additive manufacturing equipment.

\section{II.2 Description of the experimental setup}

To assess the smart-sphere performance a series of flume experiments at well-controlled and above threshold flow conditions (Figure 3 and Figure 4) have been made at one of the research flumes of the Water Engineering Lab at the University of Glasgow [9]. During these experiments, the response of the smart sphere for a range of 5 different flow rates, 4 different smart sphere sphere densities and 2-bed surface rough nesses were assessed with satisfactory results, as detailed in the following sections: The observations of this experiment were obtained from tests carried out using a Flume with the following 
channel specifications: 12 meters long, 0.4 meters deep and 0.9 meters wide. Flume walls comprise of smooth transparent glass, which enable observing sphere transport from the side. In order to preciously create intended flow heights at reasonable flow velocities, an adjustable tailgate is located at the outlet to control the flow heights. On the sidebars at the top of the flume, a mobile carriage attached, which is used to carry the ADV and measure the flow velocities at the desired locations. Figure 4 shows general layout of the experiments used in the flume.

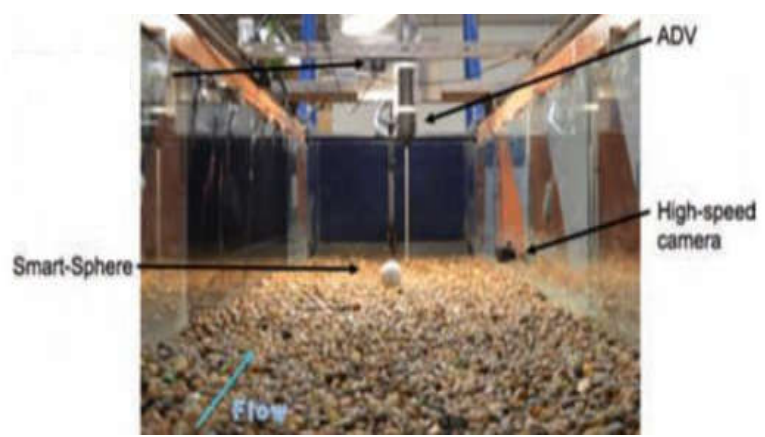

Figure 4. Test section view from upstream, showing the 'smart-sphere' resting on the flume's bed surface, the ADV for flow measurements,

Top and sidecameras for visual validation of the samrt sphere's response.

Tests were conducted using two sizes for the sphere s comprising the bed surface, over which only one 'smartsphere' was placed to be transported for each set of experiments. The nominal diameters for the bed surface sphere s during the tests are $1.5 \mathrm{~cm}$ for surface $\mathrm{A}$, and $2.15 \mathrm{~cm}$ for surface B. Both bed roughness's will have a length of $150 \mathrm{~cm}$ and width of $50 \mathrm{~cm}$ and comprise of beads in a well-packed arrangement so the bed will not move. The test section was located at the downstream end of the flume, at $5.25 \mathrm{~m}$ from the inlet, and $1.75 \mathrm{~m}$ from the tailgate. Figure 5 shows the top and side view of bed roughness $(\mathrm{A})$.

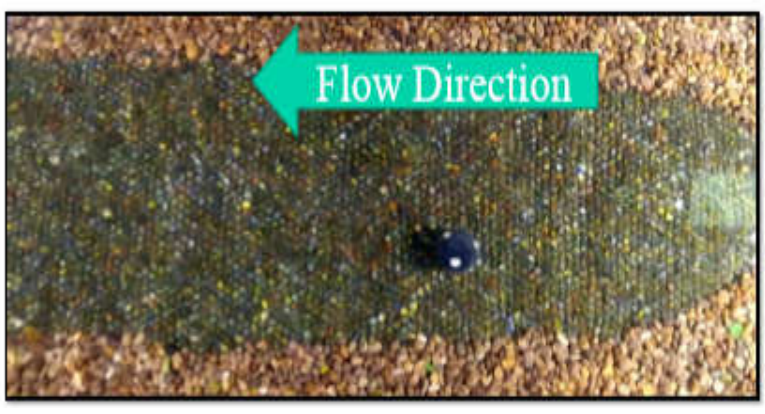

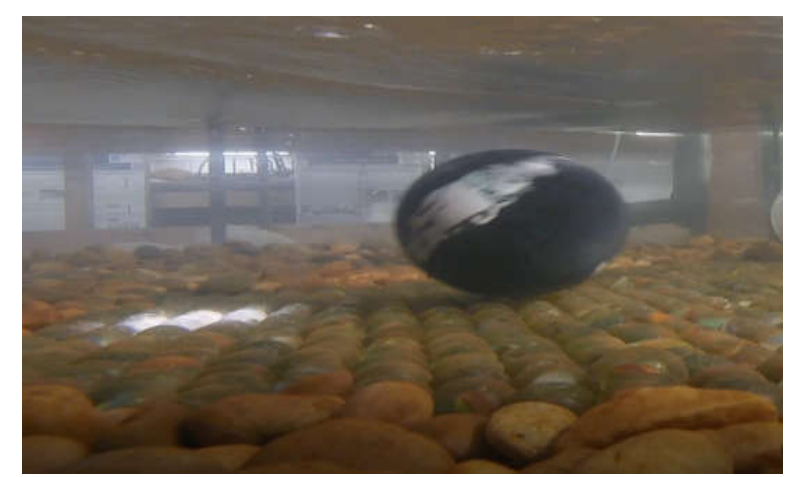

Figure 5. A) Top view of the beds rough nesses B) Side view of the bed roughness

The sphere'smotion is captured with video recording from a high-speed commercial camera to monitor and study the behavior of sphere motion by capturing the dynamical features of sediment motion. The video is used as a tracking method to capture the position and velocity of the sphere being transported downstream the flume. The camera operating at 30 frames per second (GoPro Hero4 Session), which was placed in the flume near the test section and a little bit downstream from it in order to cover the full length of the bed and to avoid any visual interference with the flow near the test section. Photolux bulbs $500 \mathrm{~W}$ were used to avoid strong light reflection on the bed and facilitate visual tracking of the sphere movements. The whole test section was viewable without any problem as shown in Figure 5. Flow velocity profiles were taken by installing an Acoustic Doppler Velocimeter in one of the mobile carriages of the flume to get flow hydrodynamics and velocity profiles. The Nortek Acoustic Doppler Velocimeter (ADV) provides time series of instantaneous flow velocity data in three directions (stream wise, vertical, and transverse), but the concerned direction was along the stream flow so the probe (sender/ receiver) of ADV was directed towards the flow direction. The stream wise velocity has the most significant amount while the other directions are extremely lower for that the stream wise velocity is only considered.

\section{II.3 Experimental protocol}

The first stage of the experiments was to visually identify the combinations of parameters for flow rates and smart sphere densities that should be set to get low sphere movement just slightly above threshold flows. The channel flow rate was increased by setting the pump of the flume to the required flow rate, and it was waited for a few minutes (3-5) to ensure fully developed steady flow conditions have reached. Flow uniformity was ensured by checking that the flow depth is the same at different distances downstream along flume and 
sufficiently high to avoid any significant water surface effects and interactions with the smart sphere. The smart sphere was marked with distinct and discernible symbols in a way to observe its orientation from 6 directions (top, bottom, and front, back, 2 sides) to enable monitoring its orientation and its movement downstream. The lead strips that were used to change sphere density were also numbered and pre-weighted to assure the sphere's weight increased in consistent way. Calibrations of the sensor such as the rotation and the vertical axis orientation were done offline, before conducting the experiments, to get reliable data. The sensor was turned on after initiating recording with video, to allow recording the time at which the sensor is turned on. After that the smart sphere was placed on the test area to monitor its transport. The camera and sensor recording were stopped after each sphere transport had ceased, at the end of the flume's test section. The flow rates, smart sphere densities and bed rough nesses were assessed as they will be changed throughout the experiment. Iterations for different densities and flow rates were 3 times for both surfaces to get the best results. Consideration was taken to ensure that each experiment started at the same relative condition, this was done by checking the water tank levels before and after operation of pump at the fixed chosen flow rate and adding any water if needed. The ADV (Figure 2) is used in the test area to record the time series of the instantaneous flow velocityin three directions (stream wise, vertical, and transverse), but the concerned direction was along the stream low so the probe (sender/ receiver) of ADV was directed towards the flow direction. The stream wise velocity has the most significant amount while the other directions are extremely lower for that the stream wise velocity is only considered. The probe is placed and oriented to look upstream. ADV tracking system moves up and down, the velocity profiles comprise of 7 points along the vertical axis as the probe moves up and down. So, seven points along the depth of the flow were measured by the ADV to get stream wise velocities and produce the velocity profiles. In order to ensure sufficient accuracy of the results, the measurement intervals along the vertical axis were denser near the hydraulically rough boundary. Each point measurement record is at least 4 minutes long. The sampling rate of $\mathrm{ADV}$ was $25 \mathrm{~Hz}$, which means the ADV measured 25 samples per second, then calculated their mean. Data acquisition and processing was done automatically by the ADV along with Matlab software for data processing. Figure 6 shows the ADV measurement points with the smart sphere resting in front of it.

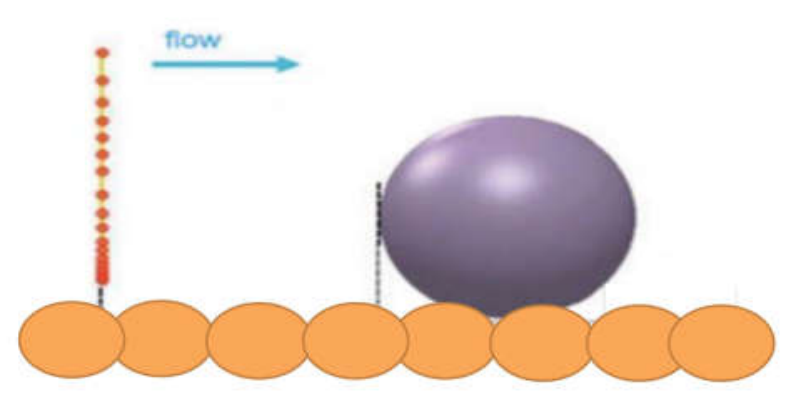

Figure 6. Side view of the smart sphere resting on the bed with the ADV used for velocity profiles measurements

\section{Results and discussion}

This section presents methods used to analyse the collected data and the preliminary results from the experimental procedure. Flow velocity profles were produced from the ADV data to relate the flow hydrodynamics (cause) to the sphere response (outcome), whereas the video recording allowed a qualitative analysis of the wide range of sphere's dynamical responses. These responses were further measured using the high frequency inertial measurements recorded by the "smart-sphere" (including angular velocities and linear accelerations), these measurements can be related to the near bed hydrodynamic forces acting on it and above threshold flow conditions.

\section{III.1 Hydrodynamic data}

Flow velocity profiles were attained at a distance of one diameter upstream the sphere's face. The profiles comprise of seven points calculated from the channel bed along at depths of $5 \mathrm{~mm}, 10 \mathrm{~mm}, 16 \mathrm{~mm}, 24 \mathrm{~mm}, 32 \mathrm{~mm}$, $42 \mathrm{~mm}, 50 \mathrm{~mm}$. These points were chosen to be as close to the bed surface, so as the capture the hydrodynamic forcing on the sphere and allow estimating bed shear stresses. For each point, time series of the flow velocity were recorded for 4 minutes and the results were postprocessed to get the average velocity. As predicted, higher flow rates have higher velocities and near the hydraulically rough boundary, the averaged velocity profiles follow closely the logarithmic shape.

\section{III.2 Analysis of video recording}

To qualitatively evaluate the sphere's responses to the hydrodynamic forces the video recordings were reviewed in slow motion. Weak sphere movements were observed for lower flow rates, while for higher flow rates the sphere movements were faster and more energetic (corresponding to greater flow and sphere Reynolds 
numbers). Clearly from Figure 7, the measured velocities over depth increase normally with higher depths for the flow rate, the water velocity increased from $18 \mathrm{~m} / \mathrm{s}$ to 23 $\mathrm{m} / \mathrm{s}$ when the water depth measured is increased from 15 to $40 \mathrm{~mm}$ for the same flow rate of $1.9 \mathrm{~m} / \mathrm{s}$. In addition, the measured velocities over depth increase with higher flow rate for the same water depth, the measured velcoity increased from 0.17 to $0.21 \mathrm{~m} / \mathrm{s}$ when the flow rate increased from 1.47 to $2.12 \mathrm{~m} / \mathrm{s}$ for water depth of 25 $\mathrm{mm}$. The velocity profiles have closely a logarithmic shape near the hydraulically rough boundary .

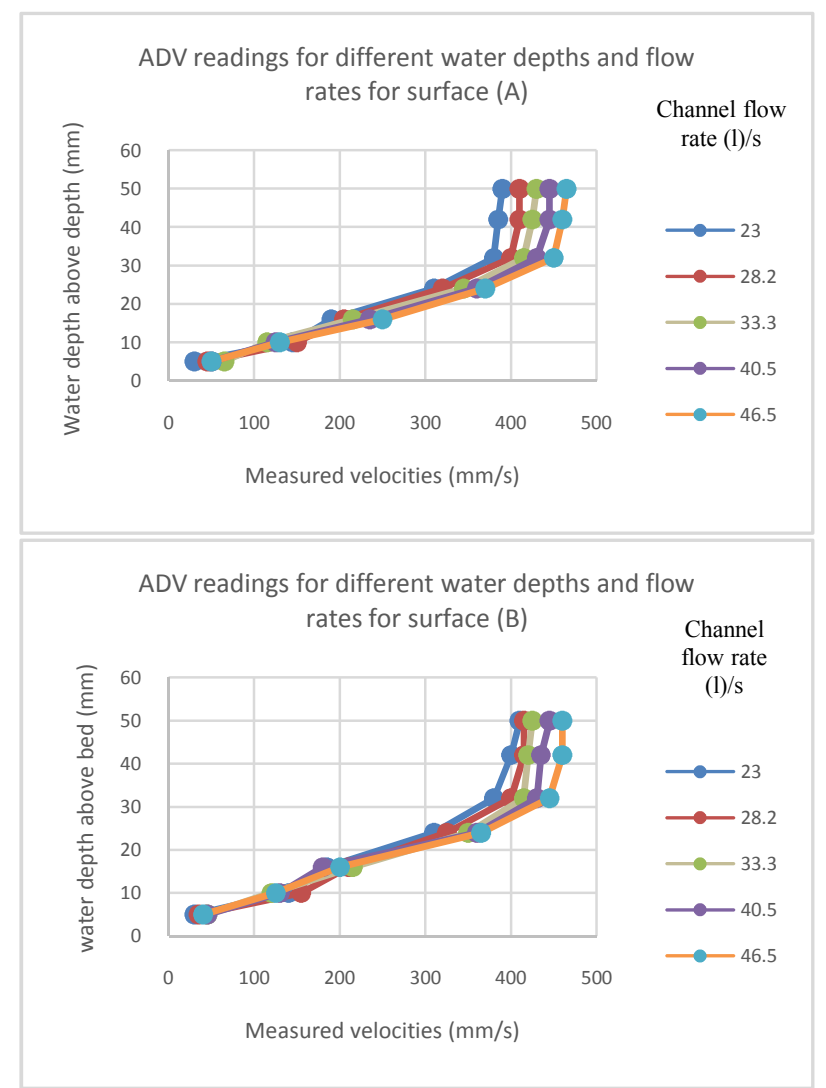

Figure 7. Plot of the time-averaged stream wise velocity profiles for different water depths and flow rates for surfaces (A) and (B)

\section{III.3 Quantitative input}

After the calibration of the smart sphere and removing the errors by using filtering and moving averages techniques. The performance of the smart sphere was assessed by comparing its rotation and acceleration readings from the sensors to the video recordings of both top and underwater high-speed camera for a range of flow rates and sphere densities. So the sphere velocity can be compared between Lagrangian and Eularian frame and by using the data from the inertial sensor and the video from the camera, the instantaneous sphere kinetic energy can be measured to understand the effect of different flow rates on the dynamics of sediment and bed energy interactions.

\section{Conclusion}

This study details the introductory results and observations from a series of designed experiments in a flume above threshold conditions. Using results from sets of sediment transport experiments with varying flow rates and sphere density, the probability distribution functions (PDFs) of sphere transport features, such as instantaneous sphere velocities, can be generated.

The objectives of this research are to employ a novel instrumented sphere, namely 'smart-sphere'to provide a better understanding of the coarse sediment transport processes at above threshold conditions,test the smart sphere under a range of well controlled flow conditions and to calibrate its response to different near bed hydrodynamic forcing.

\section{Reference}

[1] D. Sear, M. Lee, M. Collins, P. Carling, "The Intelligent Pebble: A New Technology for Tracking Particle Movements in Fluvial and Littoral Environments. In Proceedings of Erosion and Sediment Transport Measurement: Technological and Methodological Advances Workshop", Oslo, Norway, 2002, pp. 19-21.

[2] M. Spazzapan, C J. Petrovci , S. Miko ", " New tracer for monitoring dynamics of sediment " transport in turbulent flows", ActaHydrotech. 2004, pp.135-148.

[3] D.K. Abeywardana, A.P. Hu, N. Kularatna, "IPT Charged Wireless Sensor Module for River Sedimentation Detection", IEEE Sensors Applications Symposium (SAS 2012), France, 2012, pp. 1-5.

[4] M. Valyrakis, E. Pavlovskis, "Smart-pebble" design for environmental monitoring applications", 11th Int. Conf. Hydroinformatics 2014, Hamburg, Germany, 2014, pp.4.

[5] M. Valyrakis, P. Diplas, C.L. Dancey, "Entrainment of coarse particles in turbulent flows: an energy approach". Journal of Geophysical Research, 2013.

[6] P. Diplas, C. L. Dancey, A. O. Celik, M. Valyrakis, K. Greer, T. Akar, "The role of impulse on the initiation of particle movement under turbulent flow conditions". Science, Vol. 322, 2008, pp. 717-720.

[7] R. Wilson,K. M.Brook, "Miniaturized Wireless Sensor Node for Earthquake Simulation and Monitoring Applications", 7thIEEE International Symposium on Industrial Embedded Systems (SIES'12), 2012.

[8] G. Maniatis, T. Hoey, J. Sventek, "Sensor enclosures: example application and implications for data coherence". Journal of Sensor and Actuator Networks, Vol 2, 2013, pp. 761- 779.

[9] M. Valyrakis, A. Alexakis. Development of a "smartpebble" for tracking sediment transport. International Conference on Fluvial Hydraulics River Flow 2016, St. Liouis, MO, 2016, pp. 8 . 